\title{
Autoimmune Hepatitis and Sarcoidosis
}

\author{
${ }^{1}$ Shoaib Z J unejo, ${ }^{2}$ Sandeep Tuli
}

\begin{abstract}
Background: Sarcoidosis is a systemic disorder of unknown etiology. It affects the organ systems in the body, and is characterized by the presence of noncaseating granulomas in the involved organs. The most common manifestation is bilateral hilar adenopathy along with skin, joint, and eye lesions. Here, we discuss an unusual manifestation of sarcoidosis along with autoimmune hepatitis (AlH).
\end{abstract}

Case report: We report the case of a 33-year-old African American female presented with two-day history of progressive jaundice and deranged Liver function test (LFTs) the autoimmune work up was positive for anti-nuclear antibody (ANA) 1:640 and anti-smooth muscle antibody (SMA) 1:160, The serum Angiotensin converting enzyme (ACE) level was elevated 299 U/L; a right upper quadrant ultrasound revealed a mildly enlarged liver, subsequent CT chest and CT abdomen enlarged paratracheal and subcarinal adenopathy periportal edema in the liver and numerous small hypodense nodules in the spleen. Subsequent liver biopsy showed chronic, non-granulomatous, portal and parenchymal inflammation that was compatible with Autoimmune Hepatitis. Bronchoscopy with transbroncial biopsy revealed chronic, non-caseating granulomatous inflammation that was consistence with sarcoidosis. Thus the patient was diagnosed with sarcoidosis and $\mathrm{AlH}$. The patient was started on Prednisone $60 \mathrm{mg}$ daily, with subsequent reduction to 15 $\mathrm{mg}$. The serum transaminase levels returned to normal level within three months.

Conclusion: This case underscores the significance of association of Sarcoidosis that presented with Autoimmune Hepatitis. Physicians should be aware of this possibility so they may efficiently pick up the diagnosis after relevant investigations and start appropriate management. Timely intervention is key to treat the acute presentation and to prevent the further complications.

Keywords: Angiotensin-converting enzyme, Antismooth muscle antibody, Autoimmune hepatitis, Liver function test.

How to cite this article: Junejo SZ, Tuli S. Autoimmune Hepatitis and Sarcoidosis. Int J Recent Surg Med Sci 2017;3(2):124-126.

\section{Source of support: Nil}

Conflict of interest: None

${ }^{1}$ Resident, ${ }^{2}$ Attendent

${ }^{1}$ Department of Internal Medicine, Mount Sinai, Jamaica, New York, USA

${ }^{2}$ Department of Radiology, Queen Hospital Center, Jamaica New York, USA

Corresponding Author: Shoaib Z Junejo, Resident, Department of Internal Medicine, Mount Sinai, Jamaica, New York, USA Phone: +3474224189, e-mail: shoaibzahoor@gmail.com

\section{INTRODUCTION}

Sarcoidosis is a progressive multiorgan disease of unknown etiology. The prevalence of sarcoidosis in the United States is reported to be 1 per 100,000 with a three-fold higher risk in African-Americans when compared with Caucasians. ${ }^{1}$ It is characterized by the accumulation of T lymphocytes, mononuclear phagocytes, and noncaseating granulomas in involved tissues. The lung and lymph nodes are involved in more than $90 \%$ of cases, but it can involve any organ system. The liver is the third most commonly involved organ after the lymph nodes and the lungs. Sarcoidosis has been reported infrequently in association with $\mathrm{AIH}{ }^{2}$ We present a rare case of a patient with this disorder.

\section{CASE REPORT}

A 33-year-old African-American female presented with a two-day history of progressive jaundice. Liver function test (LFT) showed elevated aminotransferase that was 35 times the normal level. Due to abnormal liver-related tests, laboratory evaluation of infectious and autoimmune process was done. Her serum was positive for ANA 1:640 and SMA 1:160. No evidence of viral infections was found. The serum ACE level was elevated at $299 \mathrm{U} / \mathrm{L}$; a right upper quadrant ultrasound was obtained for abnormal LFTs. It revealed a mildly enlarged liver with increased echogenicity of portal vein radicals and decreased echogenicity of the liver parenchyma resulting in a "starry night" appearance (Fig. 1). The gallbladder wall was slightly thickened without shadowing gallstones. The common duct was not dilated. Given the nonspecific findings on ultrasound and laboratory evidence, subsequent computed tomography (CT) of chest and CT abdomen were performed with intentions of getting a liver biopsy. The CT chest demonstrated enlarged paratracheal and subcarinal adenopathy and bilateral subcentimeter hilar nodes (Fig. 2). The CT abdomen revealed periportal edema in the liver and numerous small hypodense nodules in the spleen (Fig. 3). Subsequent liver biopsy showed chronic, nongranulomatous, portal and parenchymal inflammation that was compatible with AIH. Subsequent bronchoscopy with transbronchial biopsy revealed chronic, noncaseating granulomatous inflammation that was consistent with sarcoidosis. Thus, the patient was diagnosed with sarcoidosis and AIH. The patient was started on Prednisone $60 \mathrm{mg}$ daily, with subsequent reduction to $15 \mathrm{mg}$. The serum transaminase levels returned to normal level within 3 months. 

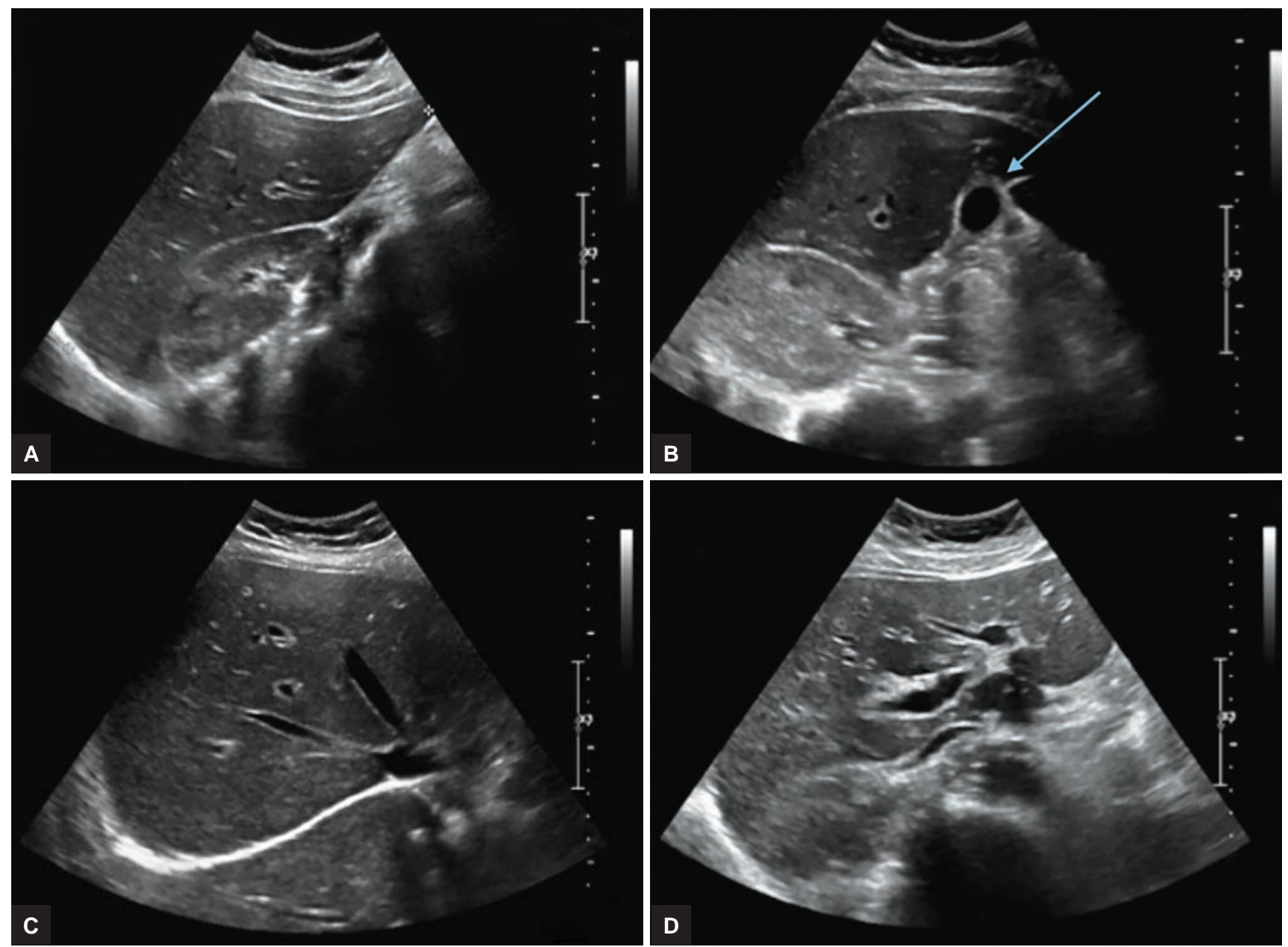

Figs 1A to D: Selected grayscale ultrasound images of the liver reveal increased echogenicity of portal vein radical walls and decreased echogenicity of the liver, giving a "starry night pattern." The liver is enlarged and gallbladder wall is thickened, a nonspecific finding (arrow)
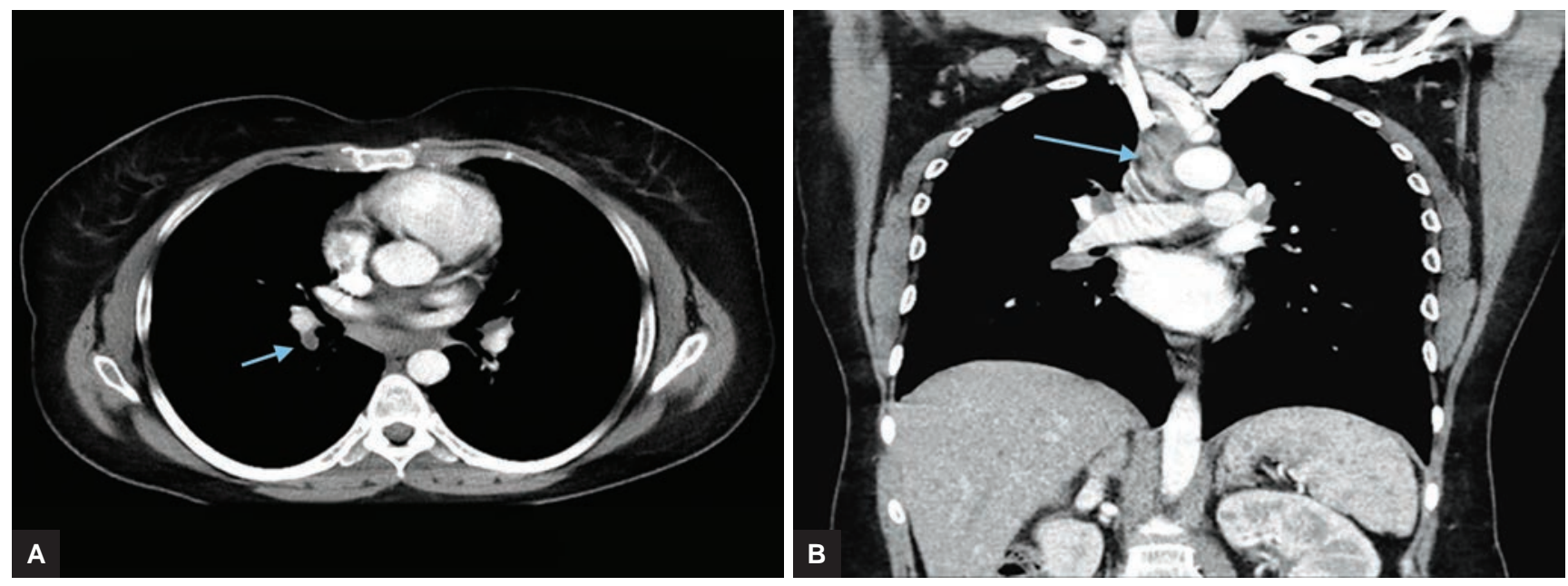

Figs 2A and B: Contrast-enhanced CT chest [(A) axial and (B) CT images] demonstrate bilateral subcentimeter hillar (short arrow) and enlarged right paratracheal (long arrow) and subcarinal adenopathy

\section{DISCUSSION}

Here, we discuss a rare presentation of sarcoidosis and $\mathrm{AIH}$. The AIH is defined as chronic hepatitis of unknown etiology. It is characterized by the presence of SMA $35 \%$ and/or ANA $15 \% .{ }^{3}$ Approximately $20 \%$ of patients with sarcoidosis of liver have hepatomegaly on physical examination. Abdominal CT scan shows homogeneous appearance of liver; hypoattenuating nodules are rarely seen. ${ }^{4}$ Liver biopsy is an important step to assess the etiology of transaminitis. In our case, the diagnosis of AIH was based on abnormal LFTs, positive SMA, and the histological findings of the liver. 


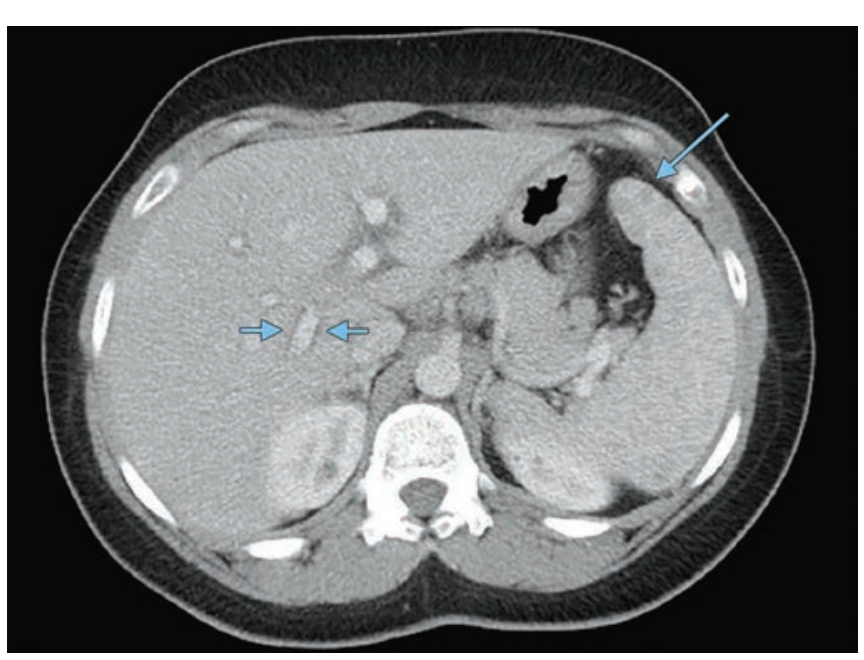

Figs 3: Contrast-enhanced axial CT image of upper abdomen demonstrates numerous subcentimeter hypodense nodules in the spleen (long arrow). Visualized portions of liver reveal low density around the portal veins (short arrows) that suggests periportal edema

The LFTs in patients with sarcoidosis include elevated aminotransaminases and granulomas on liver biopsy. ${ }^{5}$ In our case, it is possible that there were sampling errors in the liver biopsy, which did not reveal any granulomatous inflammation. However, the presence of elevated aminotransaminases, high levels of ACE, mediastinal and hilar lymphadenopathy on CT scan, and chronic granulomatous inflammation on transbronchial lung biopsy was in favor of sarcoidosis.

\section{CONCLUSION}

In conclusion, we have reported a patient with sarcoidosis and AIH. She responded well to corticosteroid therapy and achieved improvements in both sarcoidosis and liver dysfunction. The association between these two conditions along with the presence of autoantibodies can be linked to complex immunological mechanisms. Our case is unique as there has been no article on such a combination of involvement in the literature.

\section{ACKNOWLEDGMENT}

Authors would like to thank Deborah Goss, MLS (Director, Health Sciences Library, Queens Hospital Center) who reviewed and edited the manuscript.

\section{REFERENCES}

1. Newman LS, Rose CS, Maier LA. Sarcoidosis. N Engl J Med 1997 Apr;336(17):1224-1234.

2. Tojo J, Ohira H, Abe K, Yokokawa J, Takiguchi J, Rai T, shishido S, Sato Y, Kasukawa R. Autoimmune hepatitis accompanied by systemic lupus erythematosus. Intern Med 2004 Mar;43(3):258-262.

3. Czaja AJ, Nishioka M, Morshed SA, Hachiya T. Patterns of nuclear immunofluorescence in autoimmune hepatitis. Gastroenterology 1994 Jul;107(1):200-207.

4. Warshauer DM, Lee JKT. Imaging manifestations of abdominal sarcoidosis. Am J Roentgenol 2004 Jan;182(1):15-28.

5. Valla DC, Benhamou JP. Hepatic granuloma and hepatic sarcoidosis. Clin Liver Dis 2000 Feb;4(1):269-285. 